\title{
New data on old novae
}

\section{Irma Fuentes Morales*}

Universidad de Valparaíso, Chile

E-mail: irma.fuentes@uv.cl

\section{Claus Tappert}

Universidad de Valparaíso, Chile

E-mail: claus.tappert@uv.cl

\section{Vanessa Gotta}

Universidad de Valparaíso, Chile

E-mail: vanita.vane@gmail.com

\section{Nikolaus Vogt}

Universidad de Valparaíso, Chile

E-mail: nikolaus.vogt@uv.cl

\section{Alessandro Ederoclite}

Centro de Estudios de la Física del Cosmos de Aragón, Spain

E-mail: aederoclecefca.es

\section{Linda Schmidtobreick}

European Southern Observatory, Chile

E-mail: lschmidteeso.org

The role of the nova eruption within the evolution of cataclysmic variables can be studied by comparing the population of post-nova systems with the general cataclysmic variables population. However the current sample of post-novae is not statistically significant. In our project, we use photometry and spectroscopy in the optical to increase the sample of known post-novae. We here present a sample of our latest results on the objects EL Aql, V368 Sct, V1229 Aql, DY Pup and X Cir.

The Golden Age of Cataclysmic Variables and Related Objects - III

7-12 September 2015

Palermo, Italy

\footnotetext{
*Speaker.
} 


\section{Motivation}

Because the known total sample of post-novae is not large enough to perform a significant statistical analysis, in 2009 Tappert and his collaborators started a systematic search of old nova systems based on [2]'s catalogue in order to increase the sample. Systems that erupted before 1986 were selected as target/candidates. (see [9] and [4] of this volume for more details). Post-novae recovered until now within this project were published in [10], [12] and [13].

In this work we show a preliminary analysis of the recovered post-novae El Aql, V1229 Aql and V368 Sct. Additionally, we present time-resolved photometry in the optical for X Cir and DY Pup from which we estimated their orbital periods. The final analysis of those objects will be published in an oncoming paper.

\section{New post-novae confirmed from spectroscopic data}

We obtained spectroscopy of 8 post-novae in service mode using the Gemini multi-object spectrograph (GMOS) mounted at the $8.1 \mathrm{~m}$ Gemini-South telescope. The observations were performed during the first semester of 2015. GMOS has a wavelength coverage of 4200-7000 $\AA$ with a spectral resolution of $7 \AA$, thus covering efficiently the Balmer, Bowen and He lines. For each target we obtained a total of three spectra. The reduction and calibration of the data was made using the IRAF Ureka package. The spectra were corrected for the instrumental response function but not flux calibrated nor corrected for interstellar reddening.

\section{EL Aql}

EL Aql underwent a nova eruption in 1927. Currently the system shows exceptionally strong Balmer emission lines. The equivalent width for $\mathrm{H} \alpha$ is $\sim 50 \AA$ (Figure 1). This fact point outs that EL Aql has a lower mass-transfer rate than expected for an old nova. This phenomenon is also seen in V728 Sco, an eclipsing old nova recovered and analysed by [11]. Detecting such exceptional systems as EL Aql or V728 Sco is of great importance due to allow us to test the expected behaviour of the mass-transfer rate after the nova eruptions.

The He II emission line for EL Aql is very strong. At the same time the lower excitation lines like the Balmer series and $\mathrm{He}$ I are also of considerable strength. Thus it is more likely that $\mathrm{He}$ II is caused by magnetic accretion of the white dwarf rather than by irradiation in a high mass-transfer system. Therefore, we suggest that EL Aql is a magnetic CV. We furthermore believe that EL Aql has a short orbital period and a high inclination of the system, because the $\mathrm{H} \alpha$ line profile show significant shifts among individual spectra.

\section{V1229 Aql}

V1229 Aql erupted in 1970 and was confirmed as a post-nova by [3]. In 1992 [1] obtained a high resolution spectrum of this system. These authors used $\mathrm{H} \alpha-[\mathrm{N}$ II] blend to calculate the angular expansion rate for the nova shell remnant, from which they derived the distance $d=2100 \pm 900 \mathrm{pc}$. Our three low resolution spectra, taken 23 years later, still show emission from the shell remnant, which can be easily visible in the 2D image (see left side of Figure 2). In our average spectrum we see that the intensity of the $\mathrm{H} \alpha-[\mathrm{N} \mathrm{II}]$ emission line blend is lower than that observed in 1992 by 


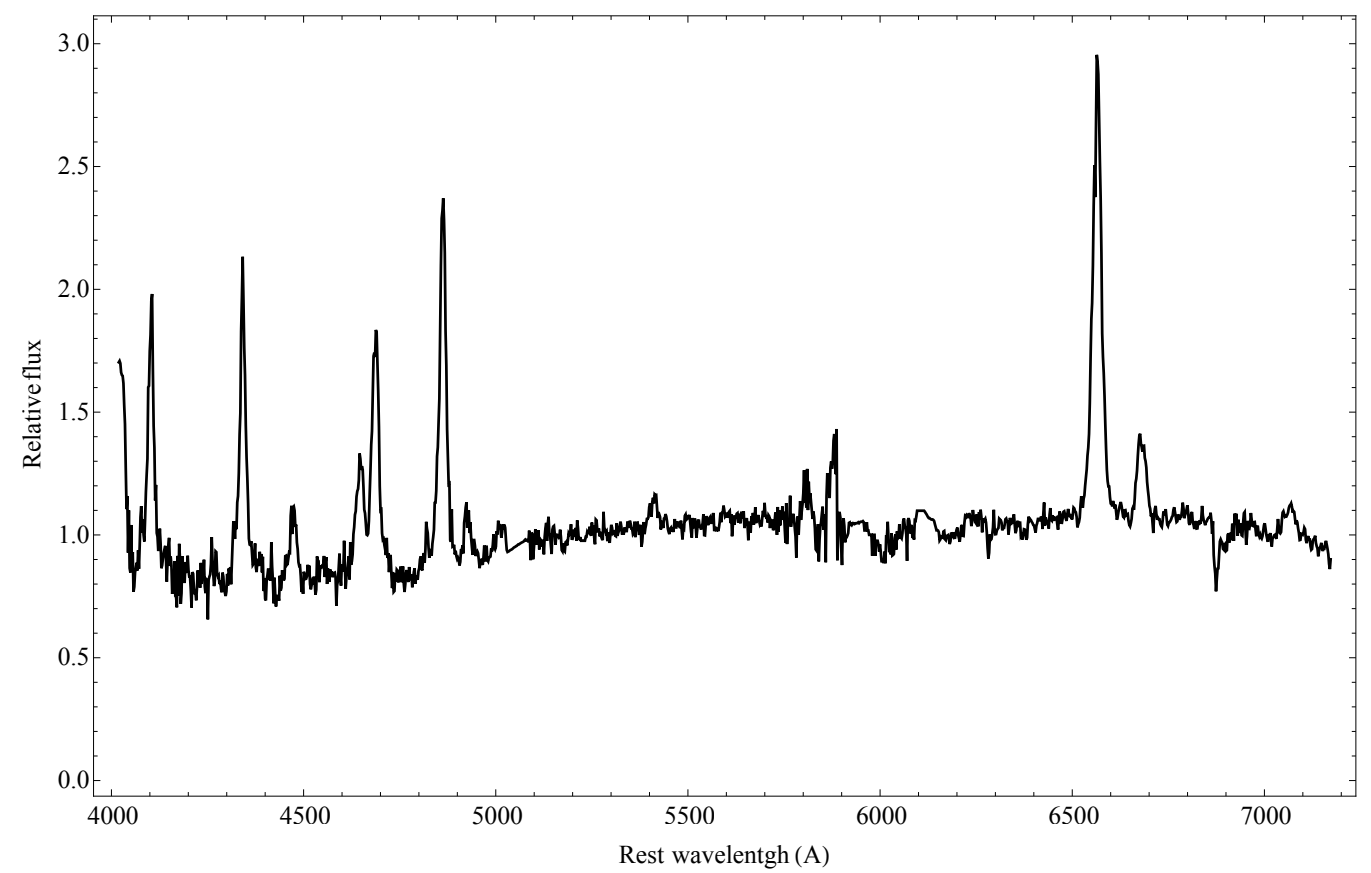

Figure 1: Low-resolution spectrum of the recovered post-nova El Aql in which He II 5412 and C IV 5812 emission lines are also present.

[1], but still present. When the high emission coming from the shell dominates in the spectrum, emission lines coming from the disc are hidden by the emission from the shell. Once the shell gets fainter we start to see features in the spectrum that are typical for the disc, e.g. He I. In the case of V1229 Aql, we can identify this line in the spectrum taken in 2015. We can roughly estimate the distance, using the radial velocity of the blue/red peaks of $\mathrm{H} \alpha$ and the size of the shell in the 2D image, as $d \sim 3 \mathrm{kpc}$, which is within the uncertainty of the [1] estimate.

The $\mathrm{H} \alpha$ profile shifts significantly in each individual spectrum over $45 \mathrm{~min}$, suggesting a short orbital period and high inclination.

\section{V368 Sct}

V368 Sct erupted the same year as V1229 Aql, and also here the nova shell is still visible after 45 years. However, the shell is fainter than the shell of V1229 Aql (Figure 2 on the right). Our rough estimate of the distance is $d \sim 760 \pm 40$ pc. Differently to V1229 Aql, we do not see variation in the $\mathrm{H} \alpha$ profile so we can not speculate about its inclination nor its orbital period.

\section{Orbital periods from optical photometry}

In 2013 and 2015 we obtained time-series photometry in V band for ten post-novae. We used direct CCD imaging at the $2.5 \mathrm{~m}$ Dupont at Las Campanas Observatory (LCO). We found the orbital period $\left(P_{\text {orb }}\right)$ for four novae. Here we describe the preliminary analysis for two of those, DY Pup and X Cir, which are eclipsing systems. Reduction and analysis was obtained with the IRAF daophot package. 

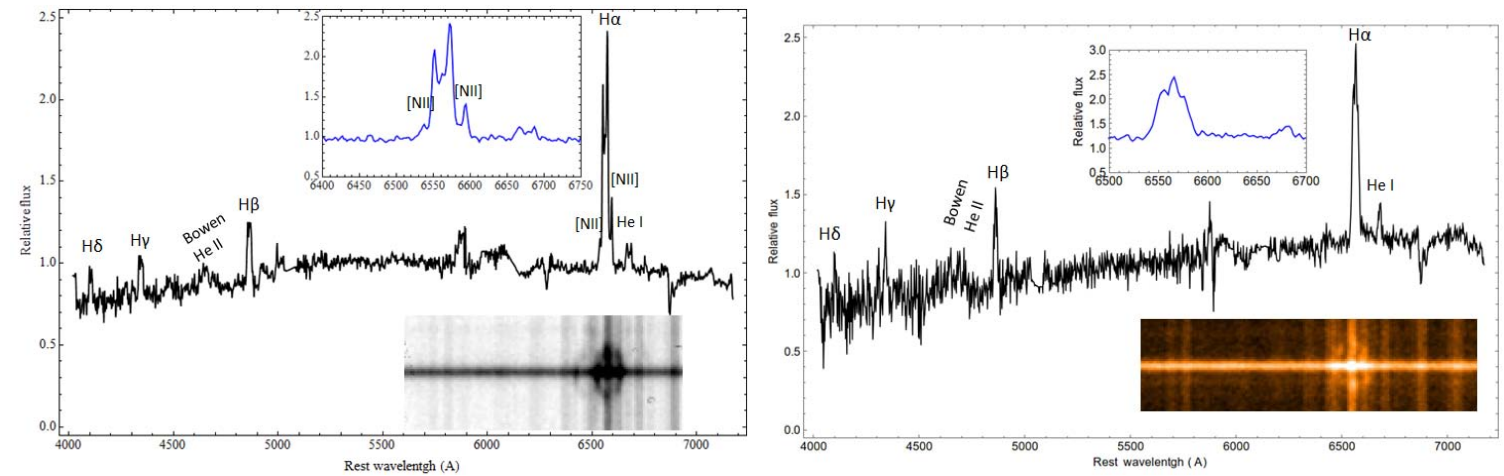

Figure 2: Low-resolution average spectra of the old novae V1229 Aql (left) and V368 Sct (right). Both systems show flux contribution coming from the shell. A close-up on $\mathrm{H} \alpha$ profile of each spectrum is shown in the inset. For both objects the shell contribution is also visible in the $2 \mathrm{D}$ image.

\section{DY Pup}

DY Pup had a eruption in 1902 with a large amplitude ( $\sim 13$ mag). Our observation covers three eclipses in total from which we derived $P_{\text {orb }}=3.34 \mathrm{~h}$. This value was already reported by Van Zyl in a poster presentation from 1999 [2], but the light curves were never published. The symmetric shape of the eclipse profile, the very small amount of flickering in the light curve and the diminished pre-eclipse hump indicates that DY Pup is a high mass-transfer CV. Because its period is within the SW Sex range ([14], [5], [6]) it is likely that it also belongs to this subclass. Additionally, the spectrum obtained by [15] shows a blue continuum and weak Balmer emission lines, supporting the high mass-transfer rate scenario.

\section{Cir}

Nova X Cir underwent an eruption in 1927. The post-nova has been recovered recently [12]. The spectral properties, in particular the double-peaked line profiles, indicate the presence of an accretion disc seen at high inclination. Like in EL Aql, the strength of the He II emission line indicates a magnetic nature of the white dwarf and the prominent Balmer emission lines are indicators of a low mass-transfer rate. From our V band photometric light curves we can see that X Cir is an eclipsing CV. We derive $P_{\text {orb }}=3.7 \mathrm{~h}$ (right plot of Figure 3 ). The light curves show strong variability outside of the eclipse. However at this stage we cannot be certain about the reality of those features because the photometry may be affected by the presence of a close visual companion. A more detailed analysis will be necessary to confirm if the variability is intrinsic.

\section{Summary}

In this work we presented a preliminary analysis of our 2015 spectroscopy obtained for the postnovae EL Aql, V1229 Aql and V368 Sct.

$\mathrm{El} \mathrm{Aql} \mathrm{is} \mathrm{likely} \mathrm{to} \mathrm{be} \mathrm{a} \mathrm{low} \mathrm{mass-transfer} \mathrm{system,} \mathrm{and} \mathrm{thus} \mathrm{does} \mathrm{not} \mathrm{follow} \mathrm{the} \mathrm{general} \mathrm{trend.}$ EL Aql is also a candidate to be a magnetic CV and a short period system.

In the case of V1229 Aql and V368 Sct, the nova shell remnant is still dominant, even 45 years after the eruption. The contribution of the shell in V368 Sct is less than in V1229 Aql. On the other 

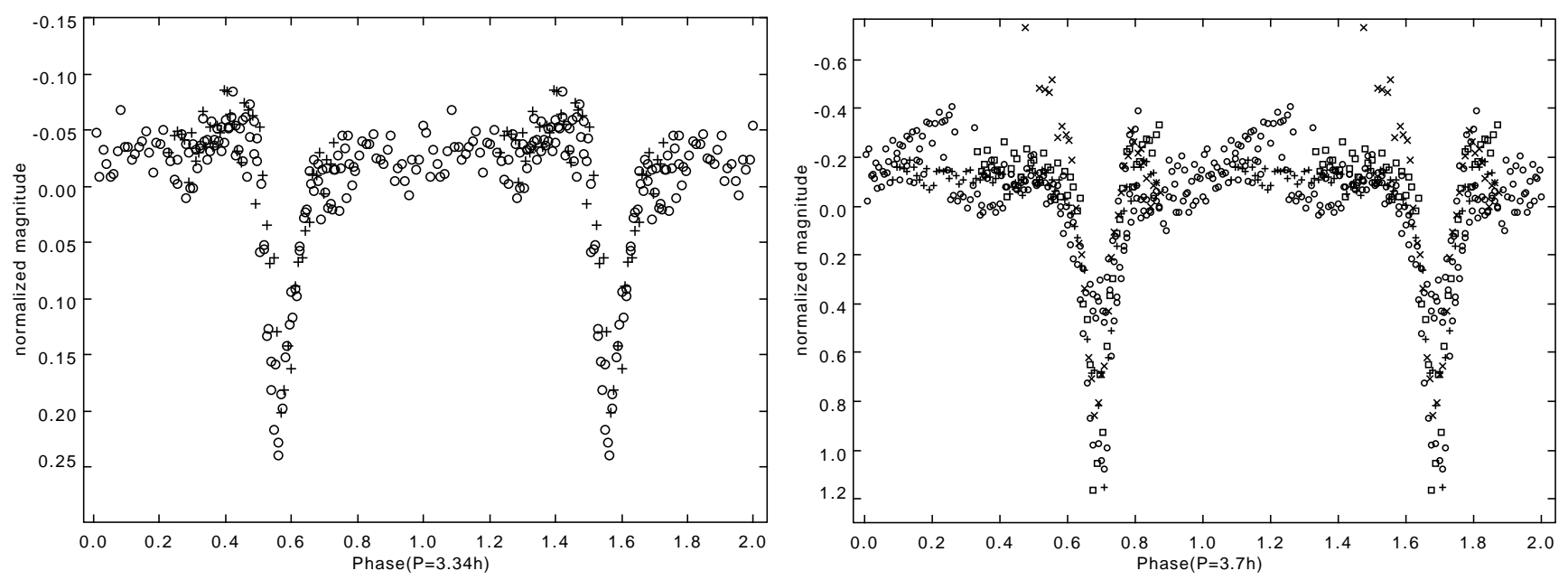

Figure 3: The normalized V magnitude vs phase using the corresponding orbital periods for DY Pup (left) and X Cir (right) are plotted into two orbital cycles. Different symbols represent different nights.

hand for V1229 Aql we find variation of the central $\mathrm{H} \alpha$ peak pointing to a short orbital period and a high inclination. We estimate a distance of $760 \pm 40 \mathrm{pc}$ and $\sim 3 \mathrm{kpc}$ for V368 Sct and V1229 Aql, respectively.

In addition we present our analysis of photometric data for the eclipsing post-novae DY Pup and X Cir. The results of DY Pup and X Cir are still rather preliminary, however we calculate orbital periods of $3.34 \mathrm{~h}$ and $3.7 \mathrm{~h}$, respectively. While DY Pup appears to be a high mass-transfer rate system, X Cir seems to be a low mass transfer system. Further studies of these systems would be desirable to derive physical parameters.

\section{Acknowledgments}

IFM thanks Odette Toloza for her comments.

This research was supported by FONDECYT Regular grant 1120338 and the Centro de Astrofísica de Valparaíso (CT, IFM and NV). AE acknowledges support by the Spanish Plan Nacional de Astrononomía y Astrofísica under grant AYA2012-30789.

\section{References}

[1] M. Della Valle and H. W. Duerbeck, Study of Nova Shells - I. - V1229-Aquilae (1970): nebular Expansion Parallax and Luminosity, A\&A 275 239-244 (1993)

[2] Ronald A. Downes, Ronald F. Webbink, Michael M. Shara, Hans Ritter, Ulrich Kolb, Hilmar W. Duerbeck, A Catalog and Atlas of Cataclysmic Variables: The Living Edition, PASP 113 764-768 (2001)

[3] Hilmar W. Duerbeck, A reference catalogue and atlas of galactic novae, SSRv 45 1-212 (1987)

[4] A. Ederoclite et al. In these proceeding

[5] P. Rodríguez-Gil, B.T. Gänsicke, H. J. Hagen et al., SW Sextantis stars: the dominant population of cataclysmic variables with orbital periods between 3 and 4h, MNRAS 377 1747-1762 (2007) 
[6] P. Rodríguez-Gil, L. Schmidtobreick and B. T. Gänsicke, Spectroscopic search for new SW Sextantis stars in the 3-4 h orbital period range - I, MNRAS 374 1359-1376 (2007)

[7] L. Schmidtobreick, In these proceeding

[8] J. V. Smoker, F. P. Keenan and A.J. Fox, The large- and small-scale Ca II K structure of the Milky Way from observations of Galactic and Magellanic sightlines, A\&A 582 59-90 (2015)

[9] C. Tappert et al. In these proceeding

[10] C. Tappert, A. Ederoclite, R. E. Mennickent, L. Schmidtobreick, N. Vogt, Life after eruption - I. Spectroscopic observations of 10 nova candidates, MNRAS 423 2476-2485 (2012)

[11] C. Tappert, N. Vogt, L. Schmidtobreick, A. Ederoclite, J. Vanderbeke, Life after eruption - II. The eclipsing old nova V728 Scorpii, MNRAS 431 92-101 (2013)

[12] C. Tappert, N. Vogt, M. Della Valle, L. Schmidtobreick, A. Ederoclite, Life after eruption - IV. Spectroscopy of 13 old novae, MNRAS 442565 -576 (2014)

[13] C. Tappert, N. Vogt, L. Schmidtobreick, A. Ederoclite, Life after eruption - V. Spectroscopy of eight candidate old novae with Gemini-South, MNRAS 450 943-955 (2015)

[14] J. R. Thorstensen, F. A. Ringwald, R. A. Wade, G. D. Schmidt, J. E. Norsworthy, PG0027 + 260 - an example of a class of cataclysmic binaries with mysterious, but consistent, behavior, AJ 102 272-283 (1991)

[15] T. Zwitter and U. Munari, CCD spectrophotometry of CVs. I. 4600-9000A low resolution atlas for 31 faint systems, A\&AS 107 503-514 (1994)

\section{DISCUSSION}

KOJI MUKAI: Do your spectra have good enough S/N to allow you to detect DIB? Perhaps this depends on the source?

IRMA FUENTES: The lines of the interstellar medium are very narrow. For instance, the equivalent widths for Ca II K and Na I D is $\sim 35-125 \mathrm{~m} \AA$ and $\sim 30-160 \mathrm{~m} \AA$, respectively (more detail in [8]). So, in order to detect it a high spectral resolution is necessary. Our spectra are low-resolution, therefore DIBs are not detected.

PABLO RODRÍGUEZ-GIL : (1) Are the spectra you are shown flux calibrated?

(2) Have you taken interstellar reddening into account?

IRMA FUENTES: (1) Our spectra are not flux calibrated yet.

(2) We have not considered yet the interstellar reddening. For this preliminary analysis it was not necessary. 